\title{
Resolving the Activation Site of Positive Regulators in Plant Phosphoenolpyruvate Carboxylase
}

Dear Editor,

Phosphoenolpyruvate carboxylase (PEPC; EC 4.1.1.31) is located at an important branch point in the carbohydrate metabolism of plants. The enzyme is a homotetramer and catalyzes the addition of bicarbonate to phosphoenolpyruvate (PEP) to form oxaloacetate and phosphate. PEPC is regulated by metabolites and phosphorylation. Allosteric feedback inhibition is mainly regulated by L-malate and L-aspartate which bind to a site separated from the active center (Kai et al., 1999; Paulus et al., 2013). Structure analysis of PEPC from Escherichia coli (Kai et al., 1999; Matsumura et al., 2002), Zea mays (Matsumura et al., 2002), Flaveria trinervia, and F. pringlei (Paulus et al., 2013) revealed that the substrate PEP and the feedback inhibitors bind to separate sites within each monomer.

Various substances such as hexose phosphates or triose phosphates as well as neutral amino acids have been identified as activators of PEPC. These substances are activating by lowering the $\mathrm{K}_{\mathrm{m}}$ for the substrate PEP (Wong and Davies, 1973; Rustin et al., 1988). Ethylene glycol (EG) is another substance activating PEPC, although neither binding site nor activation mechanism has been resolved yet. For $Z$. mays PEPC, a strong protective effect of EG at concentrations of $10-30 \%$ (v/v) has been described resulting in an about 10-fold decrease in the half-saturation concentration for PEP.

Based on enzyme kinetics and binding studies, conflicting models on the molecular mechanism causing activation of the PEPC tetramer have been proposed. In the competitive activation model supported by kinetic studies of Wong and Davies (1973), the activator binds to the active site of one subunit, induces an allosteric conformational change, and thereby activates the other subunits. Binding studies by Tovar-Méndez et al. (1998) showing mutually exclusive binding of PEP and glucose 6-phosphate (GIC-6-P) support this model. In the alternative model, the activator does not bind to the active site, but to a distinct binding site. This noncompetitive activation model is underpinned by a theoretical model of the PEPC-Glc-6-P substrate complex (Mancera and Carrington, 2005) and further supported by mutagenesis studies (Takahashi-Terada et al., 2005).

Here, we present two protein crystal structures in complex with activators. The first crystal structure has GIc-6-P bound to the active site (PDB code $4 \mathrm{BXC}$ ). In the second structure,
$4 B X H$, the active site is occupied by $E G$. The resolution $d_{\min }$ of the crystallographic data are $2.86 \AA$ for $4 \mathrm{BXC}$ and $2.24 \AA$ for $4 \mathrm{BXH}$. Based on the $\mathrm{R}_{\text {free }}$-values, the overall coordinate errors of the atoms were estimated as $0.32 \AA$ for $\triangle B X C$ and $0.17 \AA$ for $4 \mathrm{BXH}$. The crystallographic refinement statistics are given in Supplemental Table 1. Both crystals were grown in the presence of $200 \mathrm{mM}$ ammonium sulfate. The activators GIc-6-P and EG were soaked into the grown crystals (see Supplemental Methods and Supplemental Results). The PEPC structures presented here are 'dimer of dimers', with two closely binding dimers and considerably weaker tetramerization interactions (Figure 1A). During model building of $\triangle B X C$, Glc-6-P was found in the active site of the enzyme (Figure 1B). Other solutes from the crystallization solution and cryoprotectants were also found in the structure. Tris and EG bind to the protein surface. One EG molecule was found in the malate/aspartate binding site. Sulfate binds to $\operatorname{Arg}^{178}$, $\operatorname{Arg}^{179}$, $\mathrm{Arg}^{226}$, and $\mathrm{Arg}^{366}$ in the strong dimerization surface. This site corresponds to the sulfate binding site identified in previously published structures (Matsumura et al., 2002; Paulus et al., 2013). Based on the structural homology of sulfate and phosphate, this site was suggested to define the GIC-6-P binding site of PEPC (Matsumura et al., 2002). Since, in our crystal conditions, Glc-6-P does not bind to the sulfate binding site, but to the active center, we conclude that the sulfate binding site is probably not the activator binding site. Specific binding is supported by a number of prominent interactions of Glc$6-P$ in the active center (Supplemental Figure 1A). Therefore, this structure strongly supports the competitive activation hypothesis.

The sulfate binding site at the dimerization interface may bind a number of oxyanions, such as sulfate or phosphate, if those are available at sufficient concentrations. The question remains whether this site has a functional role, or just happens to be a promiscuous binding site. Sulfate and phosphate are unnecessary for the protein function, so the sulfate binding site might be not essential. In an attempt

- The Author 2013. Published by the Molecular Plant Shanghai Editorial Office in association with Oxford University Press on behalf of CSPB and IPPE, SIBS, CAS.

doi:10.1093/mp/sst 130, Advance Access publication 16 September 2013

Received 1 August 2013; accepted 1 September 2013 
A

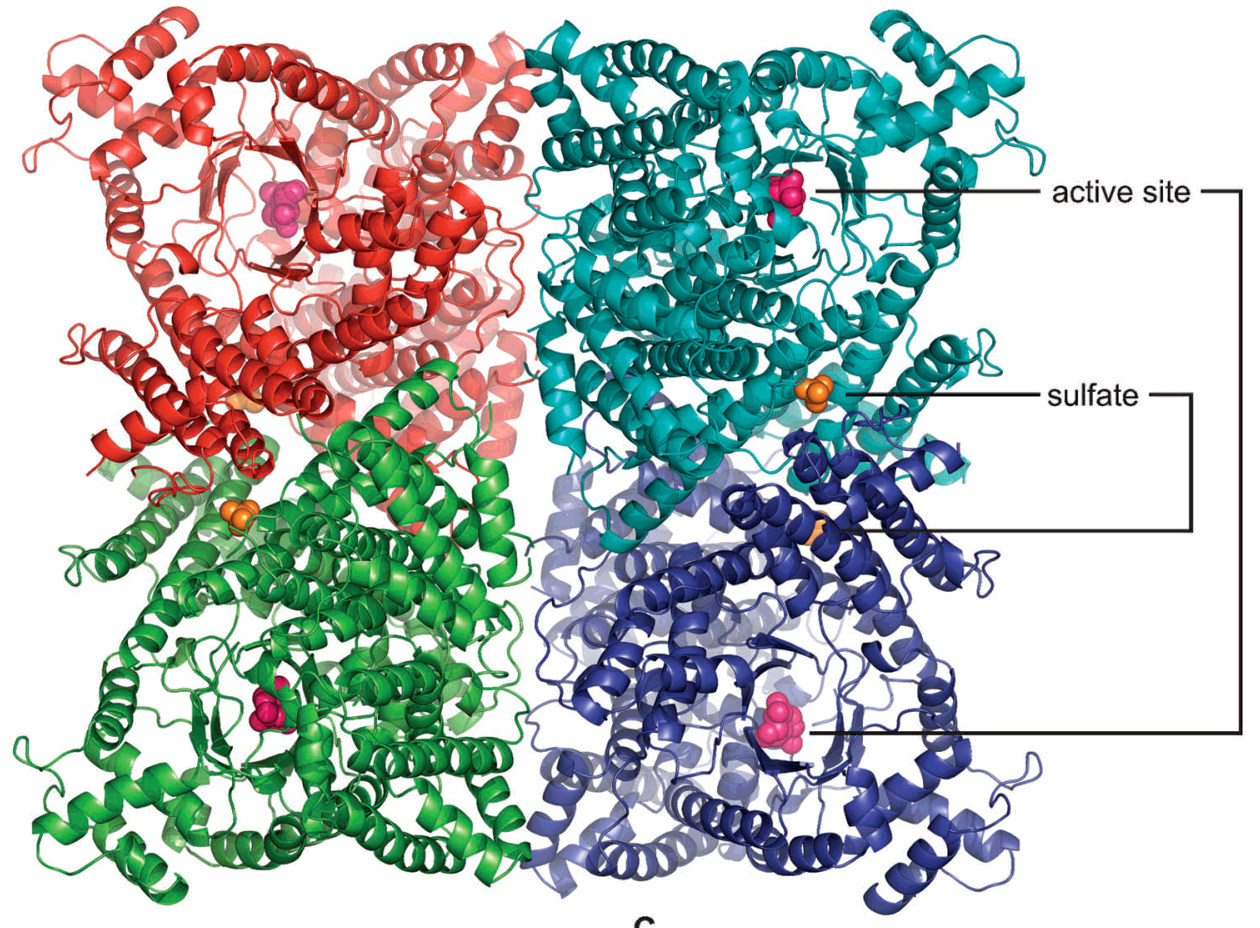

B

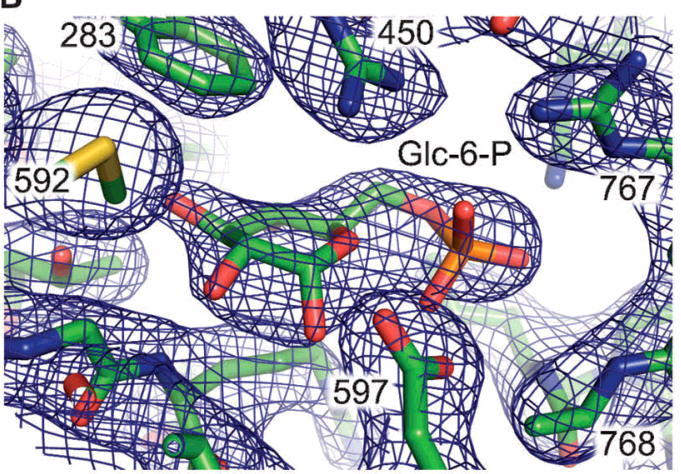

D

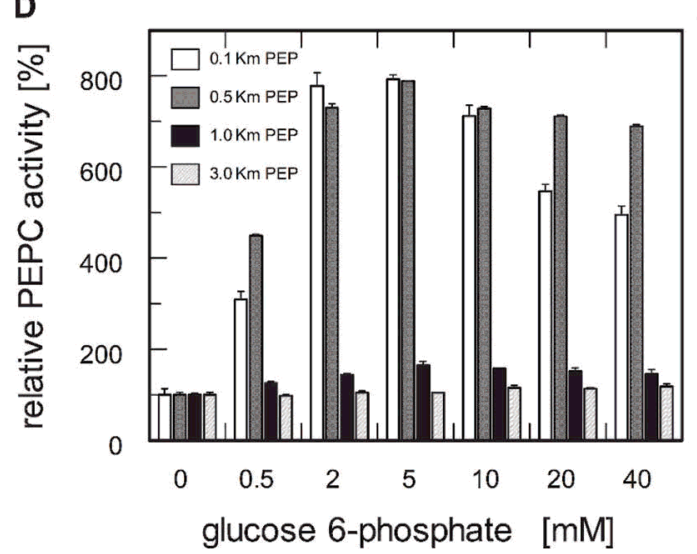

C

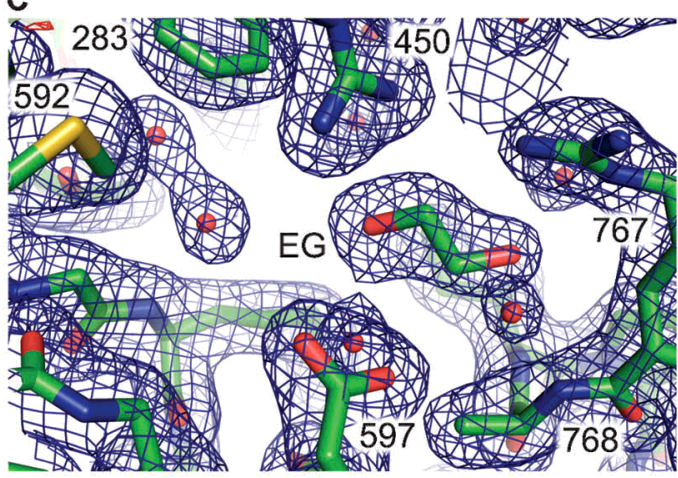

E

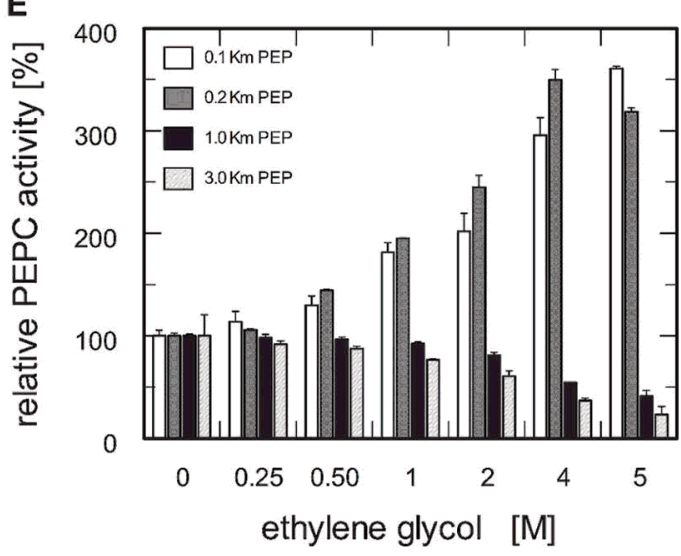

Figure 1. PEPC Binds the Activator at the Active Site, Not at the Sulfate Binding Site in the Strong Dimerization Interface.

(A) Flaveria trinervia PEPC is a 'dimer of dimers'. Two strongly interacting dimers (left and right halves of the complex) form a tetramer. X-ray crystal structure of the complex with glucose 6-phosphate (magenta) in the active sites and sulfate (orange) in the strong dimer interfaces. Glucose 6-phosphate molecules and sulfate ions are shown as spheres representing their van der Waals radii. The distance between glucose 6-phosphate and the nearest sulfate is $\sim 25 \AA$. (B, C) Glucose 6-phosphate and ethylene glycol bind to the active site of PEPC. Structures and $2 F_{o}-F_{c}$ electron densities at $1.0 \mathrm{\sigma}$. (B) Glucose 6-phosphate (Glc6-P) in the binding pocket. (C) Ethylene glycol (EG) shown from a similar point of view. The numbers refer to $\operatorname{Trp}^{283}$, Arg ${ }^{450}, \mathrm{Met}^{592}, \mathrm{Asp}^{597}$, Arg ${ }^{767}$, and $\mathrm{Arg}^{768}$. (D, E) Glucose 6-phosphate and ethylene glycol activate PEPC at low but not at high PEP concentrations. Standard deviations were calculated from triplicates. (D) The effect of glucose 6-phosphate $\left(1 \mathrm{~K}_{\mathrm{m}}\right.$ equals $0.5 \mathrm{mM}$ PEP; white, at $0.1 \mathrm{~K}_{\mathrm{m}}$; dark gray, at $0.5 \mathrm{~K}_{\mathrm{m}}$; black, at $1.0 \mathrm{~K}$; light gray, $3.0 \mathrm{~K}_{\mathrm{m}}$ ). (E) The effect of ethylene glycol on the relative PEPC activity at various PEP concentrations (white, at $0.1 \mathrm{~K}_{\mathrm{m}}$; dark gray, at $0.2 \mathrm{~K}_{\mathrm{m}}$; black, at $1.0 \mathrm{~K}_{\mathrm{m}}$; light gray, $3.0 \mathrm{~K}_{\mathrm{m}}$ ). 
to validate the theoretical model structure of Mancera and Carrington (2005), Takahashi-Terada et al. designed mutants that were supposed to prevent Glc-6-P binding and exchanged $\mathrm{Arg}^{178}, \mathrm{Arg}^{179}, \mathrm{Arg}^{226}$, and $\mathrm{Arg}^{366}$ (Flaveria numbering). The mutants effectively showed a marked decrease in Glc-6-P sensitivity or even complete desensitization (Takahashi-Terada et al., 2005). However, these mutations are located on the strong dimerization interface. Thus, the observed results could also be related to an interference of the mutants with the transmission of the allosteric signal or even a disturbance of the inter-monomeric protein contacts. Such negative impact on the enzyme function would explain the unexpected finding that the mutants are insensitive to phosphorylation of regulatory Ser ${ }^{11}$ (Ser ${ }^{15}$ in Z. mays) (Takahashi-Terada et al., 2005).

The other structure, $4 \mathrm{BXH}$, was obtained with a crystal that was just dipped in cryosolution containing $20 \%(\mathrm{v} / \mathrm{v}) \mathrm{EG} . \mathrm{EG}$, used as cryoprotectant for our crystals, was also described as activator for PEPC (Ogawa et al., 1997). Plants naturally do not contain EG, but several other polyols, such as glycerol and myo-inositol. These osmoprotectants or compatible solutes are up-regulated under environmental stresses such as drought and may have stabilizing and activating effects on PEPC similar to EG (Ogawa et al., 1997). EG binds to the periphery of the protein, but we also found it in the active site (Figure 1C and Supplemental Figure 1B). While EG was previously thought to activate PEPC by affecting global solvent properties (Ogawa et al., 1997), our structure now suggests that $E G$ acts as activator by specifically binding to the active site and, like Glc-6-P, activates competitively. Two other EG molecules are bound at the C-terminus of the protein (binding to amino acids 667 and 964-966). As with 4BXC, sulfates are bound to the previously identified sulfate binding site. The binding modes of the two activators Glc-6-P and EG are similar (Figure $1 \mathrm{~B}$ and $1 \mathrm{C}$, and Supplemental Figure 1). The protein binds G|c-6-P stronger and with more interactions than EG. The fact that Glc-6-P binds to the active site even in $20 \%(\mathrm{v} / \mathrm{v}) \mathrm{EG}$ cryosolution further emphasizes a specific binding of the Glc-6-P to the active site.

Kinetic analysis may help to discriminate whether the activators bind to the active site or somewhere else. Competitive interaction usually arises from binding to the active site (hence the name), while non-competitive interaction usually means binding to a different site. We measured the activity of PEPC at varying concentrations of Glc-6-P and EG (Figure 1D and $1 E$ ). The activation response to these substances depends on the concentration of the substrate PEP. At low concentrations of PEP, namely at the equivalent of one-tenth of the $K_{m}$ concentration, Glc-6-P and EG are strong activators. Glc-6-P activates by a factor of 8 (Figure 1D) and EG by a factor of 3.5 (Figure 1E). Optimum concentration of Glc-6-P is about $5 \mathrm{mM}$. Higher Glc-6-P concentrations result in lower activation of the enzyme. At high PEP concentrations (at $K_{m}$ or more), no distinct activation is observed. Here, Glc-6-P has no effect, while EG even acts as an inhibitor and reduces PEPC activity up to $77 \%$ (Figure 1E). These results do not support the non-competitive activation hypothesis. The only plausible explanation is a competitive binding of the activators at the substrate binding site. At low PEP concentrations, Glc-6-P and $E G$ bind to the active site and induce an allosteric activation. At concentrations above $5 \mathrm{mM}$, Glc-6-P probably occupies too many active sites of the tetramer, resulting in sub-optimal activation. At high PEP concentrations, the active sites are saturated with PEP, which is known to activate the enzyme (Rustin et al., 1988), and Glc-6-P has no effect. The inhibitory effect of EG may be the result of competitive inhibition, or due to binding of EG to the malate/aspartate inhibitor site, or a combination of both.

In summary, our kinetic and structural studies resolve the molecular basis and mechanism of positive regulation of $\mathrm{C}_{4}$ PEPC, a key player in $C_{4}$ photosynthesis. They clarify that activation of the enzyme is triggered by competitive regulation. The competitive binding model for the activation of PEPC indicated by our crystal structures of PEPC-activator complexes and kinetic studies on the $C_{4}$ PEPC from $F$, trinervia is strongly supported by previously published kinetic data (Tovar-Méndez et al., 1998; Bläsing et al., 2000).

Coordinates and structure factors have been deposited in the PDB with accession numbers $4 \mathrm{BXC}$ and $4 \mathrm{BXH}$.

\section{SUPPLEMENTARY DATA}

Supplementary Data are available at Molecular Plant Online.

\section{FUNDING}

This study was supported by the International NRW Research School BioStruct and by a grant from Deutsche Forschungsgemeinschaft through graduate program IRTG 1525 iGRAD-Plant.

\section{ACKNOWLEDGMENTS}

We thank the staff of ESRF (Grenoble) and EMBL/DESY (Hamburg) for their kind support, especially Antoine Royant and Gleb Bourenkov. We thank Elisabeth Stratmann for editorial assistance. No conflict of interest declared.

\section{Daniel Schlieper², Kerstin Förster', Judith K. Paulus, and Georg Groth'}

Institute of Biochemical Plant Physiology, Cluster of Excellence on Plant Sciences (CEPLAS), Heinrich Heine University Düsseldorf, 40204 Düsseldorf, Germany 1 To whom correspondence should be addressed. E-mail georg.groth@uniduesseldorf.de, tel. +49 211 81-12822, fax +49 211 81-13569. ${ }^{2}$ These authors contributed equally to this work.

\section{REFERENCES}

Bläsing, O.E., Westhoff, P., and Svensson, P. (2000). Evolution of the $\mathrm{C} 4$ phosphoenolpyruvate carboxylase in Flaveria, a conserved serine residue in the carboxyl-terminal part of the enzyme is a major determinant for C4-specific characteristics. J. Biol. Chem. 275, 27917-27923. 
Kai, Y., Matsumura, H., Inoue, T., Terada, K., Nagara, Y., Yoshinaga, T., Kihara, A., Tsumura, K., and Izui, K. (1999). Three-dimensional structure of phosphoenolpyruvate carboxylase: a proposed mechanism for allosteric inhibition. Proc. Natl Acad. Sci. U S A. 96, 823-828.

Mancera, R.L., and Carrington, B.J. (2005). The molecular binding interactions of inhibitors and activators of phosphoenolpyruvate carboxylase. J. Mol. Struct. Theochem. 755, 151-159.

Matsumura, H., Xie, Y., Shirakata, S., Inoue, T., Yoshinaga, T., Ueno, Y., Izui, K., and Kai, Y. (2002). Crystal structures of C4 form maize and quaternary complex of $E$. coli phosphoenolpyruvate carboxylases. Structure. 10, 1721-1730.

Ogawa, N., Kai, T., Yabuta, N., and Izui, K. (1997). Phosphoenolpyruvate carboxylase of maize leaves: an improved method for purification and reduction of the inhibitory effect of malate by ethylene glycol and bicarbonate. Plant Cell Physiol. 38, 76-80.

Paulus, J.K., Schlieper, D., and Groth, G. (2013). Greater efficiency of photosynthetic carbon fixation due to single amino-acid substitution. Nat. Commun. 4, 1518, 10.1038/ncomms2504.
Rustin, P., Meyer, C.R., and Wedding, R.T. (1988). Identification of substrate and effector binding sites of phosphoenolpyruvate carboxylase from Crassula argentea: a possible role of phosphoenolpyruvate as substrate and activator. J. Biol. Chem. 263, 17611-17614.

Takahashi-Terada, A., Kotera, M., Ohshima, K., Furumoto, T., Matsumura, H., Kai, Y., and Izui, K. (2005). Maize phosphoenolpyruvate carboxylase: mutations at the putative binding site for glucose 6-phosphate caused desensitization and abolished responsiveness to regulatory phosphorylation. J. Biol. Chem. 280, 11798-11806.

Tovar-Méndez, A., Rodríguez-Sotres, R., López-Valentín, D.M., and Muñoz-Clares, R.A. (1998). Re-examination of the roles of PEP and $\mathrm{Mg}^{2+}$ in the reaction catalysed by the phosphorylated and non-phosphorylated forms of phosphoenolpyruvate carboxylase from leaves of Zea mays: effects of the activators glucose 6-phosphate and glycine. Biochem. J. 332, 633-642.

Wong, K.F., and Davies, D.D. (1973). Regulation of phosphoenolpyruvate carboxylase of Zea mays by metabolites. Biochem. J. 131, 451-458. 